\title{
Thermal Conductivity of the Ga-In System: Experimental Measurements and CALPHAD Modeling
}

\author{
Parker Maivald ${ }^{\mathrm{a}}$, Soumya Sridar ${ }^{\mathrm{b}}$, Wei Xiong ${ }^{*}$, \\ a Shady Side Academy, 432, Fox Chapel Road, Pittsburgh, PA 15238, USA. \\ ${ }^{\mathrm{b}}$ Physical Metallurgy and Materials Design Laboratory, Department of Mechanical \\ Engineering and Materials Science, University of Pittsburgh, Pittsburgh, PA 15261, USA.
}

\begin{abstract}
Thermal interface material (TIM) that can exist as liquid at the service temperature enables efficient heat transfer across two adjacent surfaces in electronic applications. In this work, the thermal conductivities of different phase regions in the Ga-In system at various compositions and temperatures are measured for the first time. A modified comparative cut bar technique is used for the measurement of the thermal conductivities of $\operatorname{In}_{\mathrm{x}} \mathrm{Ga}_{1-\mathrm{x}}(\mathrm{x}=0,0.1,0.214,0.3$, and 0.9 ) alloys at $40,60,80$, and $100^{\circ} \mathrm{C}$ that are the temperatures commonly encountered in consumer electronics. The thermal conductivity values for the liquid and semi-liquid (liquid $+\beta$ ) Ga-In alloys are higher than the TIM currently used in consumer electronics. These measured quantities, along with the available experimental data from the literature, served as input for the thermal conductivity parameter optimization using the CALPHAD (CALculation of PHase Diagram) method for the pure elements, solution phase, and two-phase region. A set of selfconsistent parameters for the description of the thermal conductivity of the Ga-In system is obtained. There is good agreement between the measured and calculated thermal conductivity values for all the phases. Hence, it can be envisaged that liquid/semi-liquid Ga-In alloys can be considered as a potential TIM in consumer electronics due to its high thermal conductivity.
\end{abstract}

Keywords: Ga-In; thermal conductivity; CALPHAD; comparative cut bar method; thermal interface material

* Corresponding author: Tel: +1-(412) 383-8092, Tel: +1-(412) 624-4846

Email:weixiong@pitt.edu orw-xiong@outlook.com 


\section{Introduction}

For effective heat transfer by conduction, materials with high thermal conductivity such as copper are used as heat sinks in consumer electronics. Moreover, good thermal contact between the heat sink and central processing unit (CPU) surfaces are required, across which the heat transfer occurs. The use of expensive thermally conducting materials as heat sinks will not be effective in the absence of good thermal contacts, and it can be attained with the use of a thermal interface material (TIM) [1]. At present, the increasing power and reduced die size of CPUs used in computers increase the need for significantly improved TIMs. As the materials to be interfaced are good thermal conductors such as copper, the effectiveness of a TIM is enhanced by high thermal conductivity. In addition, the low thickness of the interface material, as well as low thermal contact resistance between the interface material and each mating surface, are also required [2]. Since the mating surfaces are not perfectly smooth, the interface material must be able to flow or deform so as to conform to the topography of the mating surfaces. Figure 1 illustrates the difference between the use of a solid TIM such as elastomeric pads and liquid TIM such as thermal greases and pastes in between the CPU and the heat sink. The solid TIM will lead to the formation of small insulating air pockets that will obstruct the heat conduction between CPU and heat sink. On the other hand, a liquid TIM will conform to the surface undulations of the mating surfaces efficiently such that there are no air packets formed between the CPU and the heat sink, hence, leading to effective conduction of heat as shown in Fig. 1.

The characteristics of an ideal TIM are high thermal conductivity, minimal thickness, nontoxic, manufacturing friendly, and can be easily deformed by small contact pressure to conform to the surface undulations in both the mating surfaces [1]. Therefore, it is evident that TIMs that can exist as the liquid phase at the service temperature $\left(40-100^{\circ} \mathrm{C}\right)$ for electronic applications are highly desirable for attaining higher heat conduction with maximum thermal contact between the mating surfaces. Besides, liquids that possess high thermal conductivity will be more suitable, and hence, liquid metals or metallic alloys can be potentially applied as TIMs. It is well known that only two metals can exist as liquid close to room temperature, namely, Mercury ( $\mathrm{Hg}$ ) and Gallium (Ga). Since $\mathrm{Hg}$ is known to be toxic, $\mathrm{Ga}$ is the obvious choice as its melting point is close to room temperature $\left(\sim 30^{\circ} \mathrm{C}\right)$. The addition of an alloying element that has high solubility with Ga allows the liquid phase to be stable for an extended composition range. Indium (In) belongs to the same group as Ga with the same valency, similar crystal structure (In: tetragonal and Ga: orthorhombic), lower melting point $\left(\sim 157^{\circ} \mathrm{C}\right)$ in 
comparison with other elements and comparable thermal conductivity. Hence, liquid Ga-In alloys can be considered as a candidate material for TIM in consumer electronics and knowledge of its thermal conductivity is imperative.

At present, thermal conductivity $(\kappa)$ of metallic alloys, especially the solid phases, are calculated from the specific heat capacity $\left(C_{p}\right)$, thermal diffusivity $(\alpha)$ and density $(\rho)$ using the following equation $[3,4]$.

$$
\kappa=\alpha \rho C_{p}
$$

The thermal diffusivity as a function of temperature and density at room temperature are measured using the laser flash method and Archimedes principle, respectively. The specific heat capacity is determined either using the Neumann-Kopp rule [3] or from equilibrium calculations with the thermodynamic database developed using the CALPHAD (CALculation of PHAse Diagrams) method as input [4]. The error associated with the measurement of thermal diffusivity using the laser-flash method is nearly $\pm 3 \%[3,4]$ and the uncertainty in the density measurements using the Archimedes principle is $\pm 5 \%$. In addition, the reliability of the calculated specific heat capacities were assessed to be less than $\pm 5 \%[3,4]$. Hence, due to the propagation of errors during the multiplication of these quantities, the total error in the thermal conductivity values calculated using Equation 1 is $\sim \pm 13 \%$. Moreover, this method has not been demonstrated to be successful for materials in the liquid state.

In order to circumvent this shortcoming, a modified comparative cut bar technique is used for the measurement of thermal conductivity of the Ga-In alloys in liquid and semi-liquid state, in this work. In the comparative cut bar technique, two materials are involved, namely the sample and the standard that are placed in series between the heat source and the heat sink [5], as shown in Fig. 2(a). The thermal conductivity of the standard material is known. This setup is heated by a heat source with known steady-state power input, and the change in temperature $(\Delta \mathrm{T})$ is measured using the temperature sensors that are placed in the sample and the standard across a given length (L) after a steady-state temperature distribution is reached. In general, either thermocouples or thermistors are used as the temperature sensors, and the error associated with the measurement of $\Delta \mathrm{T}$ will be less than $\pm 1 \%$. Since the amount of heat flow through the standard equals to the heat flow through the sample, the thermal conductivity of the sample is given by [5]

$$
\kappa_{1}=\kappa_{2} \frac{\mathrm{A}_{2} \Delta \mathrm{T}_{2} \mathrm{~L}_{2}}{\mathrm{~A}_{1} \Delta \mathrm{T}_{1} \mathrm{~L}_{1}}
$$


where the subscripts 1 and 2 denote the sample and the standard, respectively, and $\mathrm{A}$ is the surface area. Since the uncertainty arises only from the measurement of temperature change, which is less than $1 \%$, this method is accurate for the measurement of thermal conductivity. The changes to the original setup were undertaken in this work such that the process will be transient and capable of measuring the conductivity of liquid and semi-liquid alloys. The setup was modified as shown in Fig. 2(b), where the standard is in contact with half of the sample. It is assumed that the temperature at the sample/standard interface is equal to the temperature at the exposed surface of the sample. Therefore, only three temperature sensors will be required for the measurement of thermal conductivity using the modified method.

CALPHAD method [6,7] is a useful tool to establish databases for thermophysical properties [8-11]. It involves a computer-assisted modeling procedure with experimental data as input to develop a set of self-consistent parameters for the description of the thermophysical property. One of the major advantages of the CALPHAD technique is though, limited experimental data from a certain composition and temperature range are used as input for the database development, the properties of alloys in other composition and temperature ranges can be estimated. This cuts down the experimental efforts and costs drastically. In the present study, the thermal conductivities of Ga-In alloys in liquid and semi-liquid phase regions were measured using the modified comparative cut bar technique. With the available experimental data from literature and measurements from present work as input, the thermal conductivities of the Ga-In system, including the pure elements, solution phases, and two-phase regions, were evaluated using the CALPHAD method.

\section{Experimental procedure}

The Ga-In phase diagram calculated using the Gibbs energy descriptions developed by Anderson et al. [12] is shown in Fig. 3. Five $\operatorname{In}_{x} \mathrm{Ga}_{1-\mathrm{x}}$ alloys ( $\mathrm{x}=0,0.1,0.214,0.3$ and 0.9 ) were considered in this work from different phase regions namely, $\alpha$, liquid and liquid $+\beta$ regions. $\alpha$ and $\beta$ denote the Ga and In phases, respectively. There is a limited dissolution of Ga into In, whereas there is a negligible dissolution of In into Ga, as shown in Fig. 3. The thermal conductivities of these alloys were measured at four different temperatures namely, $40^{\circ} \mathrm{C}$, $60^{\circ} \mathrm{C}, 80^{\circ} \mathrm{C}$, and $100^{\circ} \mathrm{C}$ that are the temperatures commonly encountered in consumer electronics. The thermal conductivity of the $\beta$ phase could not be measured since In is solid in the temperature range of interest, and the setup was mainly designed for measuring the thermal conductivity of the liquid/semi-liquid phase. Vials of Ga (purity: 99.99\%, Fisher Scientific, 
USA) and In powder (purity: 99.99\%, Fisher Scientific, USA) were chosen as the raw materials. These alloys were prepared by simple mechanical mixing with a stainless steel spatula, as the two metals readily dissolve each other at room temperature. The potential for $\mathrm{Ni}$ and Fe contamination was considered to be negligible, as demonstrated by Prokhorenko et al. [13] that the dissolution of these metals into $\mathrm{Ga}$ is in the order of $10^{-2} \mathrm{wt} . \%$ after sustained heating at higher temperatures and time periods than those used in this work. Hence, either low alloy steel or pure iron needs to be chosen as the standard as elements other than Fe could add systematic error to the measurements by dissolving into the Ga-In alloy.

As shown in Fig. 4, the temperature of the sample, standard, and heat source were recorded using surface temperature sensors (STS, supplied by Vernier, USA) and recorded using the LoggerPro software through Vernier LabQuest Mini. One sensor was embedded into the surface of the heat source via a shallow drilled hole, and the sensor monitoring the standard was affixed using a small amount of cyanoacrylate glue. The STS that measures the surface temperature of the sample directly was lightly ground to provide a constant surface area for the area of contact. Subsequently, the sensors (and the surfaces they were affixed in/on) were calibrated using LoggerPro's calibration function against an ice bath, a $50^{\circ} \mathrm{C}$ sand bath kept in an oven (Fisherbrand Isotemp, Fisher Scientific, USA), and boiling water. Once the voltage readings from all the sensors were stabilized, the temperature of the sensors were set equal to the target temperature mentioned above. The hotplate was set to the desired final temperature, and the system was given an hour to equilibrate and allow the temperatures to stabilize, with each STS recording the temperature every 2 seconds. After the completion of each trial, a logistic fit was applied to each curve, in order to eliminate the relative temperature fluctuations and their effect on the calculation of the final thermal conductivity.

\section{Thermal conductivity models}

Based on the models developed by Zhang et al. [3], the thermal conductivity of the pure elements, solution phase and two-phase region of the Ga-In system was optimized using the CALPHAD method in the present modeling.

\subsection{Pure elements}

Thermal conductivity of the pure elements in liquid and solid states were described as a function of temperature using the following equation:

$$
\kappa_{0}=\mathrm{A}+\mathrm{BT}+\mathrm{CT}^{-1}
$$


where $\kappa_{0}$ is the thermal conductivity of the pure element, and $\mathrm{T}$ is the temperature. $\mathrm{A}, \mathrm{B}$ and $\mathrm{C}$ are the parameters that need to be optimized with the experimental data as input. The thermal conductivities of pure In and Ga in both liquid [14] and solid states [15,16] were obtained from the literature.

\subsection{Solution phase}

The thermal conductivity of the solution phase i.e., the liquid phase was described using the Redlich-Kister polynomials [17]:

$$
\kappa_{I n, G a}^{L}=x_{I n} \kappa_{I n}+x_{G a} \kappa_{G a}+x_{I n} x_{G a} \sum_{i=0}^{n}{ }^{i} \mathrm{~L}_{\mathrm{In}, \mathrm{Ga}}^{\mathrm{L}}\left(x_{\mathrm{In}}-x_{G a}\right)^{i}
$$

where, $\kappa_{I n, G a}^{L}$ is the thermal conductivity of the liquid phase in the Ga-In system, $x_{k}$ and $\kappa_{k}$ are the mole fraction and thermal conductivity of the pure element $k$, respectively. ${ }^{\mathrm{i}} \mathrm{L}_{\mathrm{In}, \mathrm{Ga}}$ is the $i^{\text {th }}$ order interaction parameter that describes the effect of solute atoms on the thermal conductivity of the phase. The interaction parameters can be considered to be linearly dependent on temperature and can be expressed as follows.

$$
{ }^{i} \mathrm{~L}_{\mathrm{In}, \mathrm{Ga}}^{\mathrm{L}}=a_{i}+b_{i} T
$$

where, $a_{i}$ and $b_{i}$ are the parameters that will be optimized with the experimental data measured for the liquid phase in this work as input.

\subsection{Two-phase region}

New interfaces that form due to the presence of the second phase will act as a source for scattering electrons. The interface scattering parameter was introduced to describe the effect of the second phase on thermal conductivity. The thermal conductivity of the two-phase region i.e., the $\mathrm{L}+\beta$ phase region of the Ga-In system, was described as follows.

$$
\kappa_{(L+\beta)}=n_{\mathrm{L}} \kappa_{\mathrm{L}}+n_{\beta} \kappa_{\beta}-n_{\mathrm{L}} n_{\beta} \sum_{j=0}^{m}{ }^{j} \mathrm{M}_{(\mathrm{L}+\beta)}\left(n_{\mathrm{L}}-n_{\beta}\right)^{j}
$$

where, $\kappa_{(L+\beta)}$ is the thermal conductivity of the alloys in the $\mathrm{L}+\beta$ two-phase region, $n_{l}$ and $\kappa_{l}(l$ $=\mathrm{L}, \beta)$ are the phase fraction and thermal conductivity of the phase $l$, respectively. ${ }^{j} \mathrm{M}_{(\mathrm{L}+\beta)}$ is the $j^{\text {th }}$ interface scattering parameter which will be considered as linearly dependent on temperature, similar to the Redlich-Kister interaction parameters for the solution phase and given as follows.

$$
{ }^{j} \mathrm{M}_{(\mathrm{L}+\beta)}=c_{j}+d_{j} T
$$


where $c_{j}$ and $d_{j}$ are the parameters that will be optimized with the experimental data measured from the two-phase region in this work, as input.

Once the parameters for the pure elements and the liquid phase were optimized, the interface scattering parameters were further assessed to fit the measured thermal conductivities for the alloys in two-phase regions. The extrapolation from the parameters that correspond to the two individual phases that comprises the two-phase region, in the combination of interface scattering parameters that can describe the thermal conductivity of the two-phase region. Since the database file (TDB) consists of the Gibbs energy and thermal conductivity parameters for the individual phases, it is difficult to include the thermal conductivity parameters for the twophase region into this file. Hence, the thermal conductivity for the individual phases are written into the TDB file, and the interface scattering parameters for the two-phase region will only be written into the macro file (TCM) in the form of an equation during the calculation.

\section{Results and Discussion}

Currently, the TIMs that are used in consumer electronics are thermally conductive elastomers, thermal grease, and phase change materials (PCMs). Thermally conductive elastomers, which is a solid TIM, require high contact pressures in order to fill the gaps in between the mating surfaces for effective conduction, and its thermal conductivity is very low $\left(\sim 1.2 \mathrm{Wm}^{-1} \mathrm{~K}^{-1}\right)[1]$. Subsequently, an improved TIM called thermal grease was introduced, which was composed of a thermally conductive filler dispersed in silicone or hydrocarbon oil to form a paste [18]. These materials exhibit high thermal performance at low contact pressures ( 12 psi) [19] and can fill the interstices of the mating surfaces efficiently. The reported thermal conductivities of the thermal greases currently available in the commercial market are between 3 and $7.5 \mathrm{Wm}^{-}$ ${ }^{1} \mathrm{~K}^{-1}[1]$. However, thermal greases are not manufacturing friendly as they are messy and difficult to apply, and the grease degradation rate is a strong function of operating temperature and number of thermal cycles [20].

PCMs are made of a mixture of suspended particles of high thermal conductivity, such as fine particles of metal oxide and base material. The name "phase change material" is a misnomer since it is not a phase but a change in viscosity occurs in this material [21]. The base material can be a natural material such as fully refined paraffin, polymer, co-polymer, or a combination of all [22]. The base material is solid at room temperature and behaves like grease after reaching the phase change temperature $\left(50-90^{\circ} \mathrm{C}\right)$. Commercially available PCMs are in the form of a compound or composite of specified thickness, and their thermal conductivity ranges between 
0.7 to $20 \mathrm{Wm}^{-1} \mathrm{~K}^{-1}[1]$. However, moderate contact pressure (10-50 psi) is required to bring contact between the mating surfaces, and the phase change properties limit the choice of base material and filler combinations [20].

The thermal conductivity values measured using the modified comparitive cut bar technique at different compositions corresponding to different phase regions of the Ga-In system at various temperatures are summarized in Table 1. It is clearly evident that the thermal conductivity of pure $\mathrm{Ga}$ in the liquid state, as well as the liquid and semi-liquid Ga-In alloys, are much higher than that of the currently available commercial TIMs. The primary criterion, i.e., the high thermal conductivity of Ga-In alloys and pure Ga to serve as a TIM has been satisfied. Both In and $\mathrm{Ga}$ are non-toxic, and simple mechanical mixing at room temperature is sufficient for producing these alloys due to their high solubility in the liquid state, thus, manufacturing friendly. Moreover, as mentioned before, as it is in the liquid/semi-liquid state, lower contact pressures will be sufficient to fill the air pockets between the mating surfaces that need to be measured further. This proves that Ga-In alloys in liquid/semi-liquid state and pure Ga in liquid state are expected to satisfy all the criterion required for an ideal TIM in consumer electronic applications.

From this study, it has also been proved that the modified comparative cut bar technique can be applied successfully for the measurement of thermal conductivities of liquid and semi-liquid metallic alloys. The original comparative cut bar technique can be used for measurement of the thermal conductivity of solid phases in place of the currently used technique that involves the measurement of thermal diffusivity and density as well as estimation of specific heat capacity. This is because the error associated with the former technique is very low $(\sim \pm 1 \%)$ which arises only from the temperature drop measurement, in comparison with the high error $(\sim \pm 13 \%)$ that arises due to the multiplication of the individual quantities (Equation 1) in the latter. At present, this measurement technique has been applied from room temperature to a maximum temperature of $100^{\circ} \mathrm{C}$ for low melting alloys such as the Ga-In system. However, with the use of improved temperature sensors that can detect higher temperatures, the modified comparative cut bar technique can possibly be employed for measuring the thermal conductivity of the liquid phase in commercial multicomponent alloys such as steels and Ni-base superalloys. Moreover, it can be observed in Fig. 3 and Table 1 that the data points for certain temperatures and compositions are missing. These measurements failed probably because of the dissolution of Fe from the steel block that increased the thermal conductivity considerably such that these values become outliers from the trend followed. Thus, it can be understood that the choice of 
the standard material is crucial, and it should be chosen such that it does not contaminate and alter the thermal conductivity values of the sample material.

The PARROT [23] module of Thermo-Calc software [24] was used for optimizing the thermal conductivity model parameters. The thermodynamic equilibrium information, such as solid solubility and mole fraction of phases, were simultaneously extracted by using the thermodynamic database for the Ga-In system [12] during the optimization of thermal conductivity. Initially, the thermal conductivities of the pure elements in liquid and solid states were optimized. The parameters A, B, and C in Equation 3 were optimized simultaneously for each phase with their corresponding experimental data used as input. All the experimental data were used for the optimization of the parameters with equal weight for pure In and Ga in both liquid and solid states. Once the parameters of the pure elements were fixed, the thermal conductivity parameters for the liquid phase in the Ga-In system were assessed to obtain the Redlich-Kister interaction parameters with the experimental data measured in this work as input. As the description for the individual phases was established, the interface scattering parameters for the $\mathrm{L}+\beta$ two-phase region were optimized to fit the measured thermal conductivities of the alloys belonging to that phase region from the present work. The thermal conductivity parameters for the individual phases and the two-phase region of the Ga-In system assessed in this work are listed in Table 2.

The calculated thermal conductivities for pure In and Ga in liquid and solid states are shown in Figs. 5 and 6 and compared with the reported experimental data from literature [Refs]. In the case of liquid Ga (Fig. 5(a)), the experimental data from literature and present work are compared with the calculated thermal conductivity values. It is evident from these figures that all the experimental data are in good correlation with the calculated values for both the pure elements in liquid and solid states. The high level of precision in the calculated results for pure elements ensures that the thermal conductivity of the liquid and two-phase region in the Ga-In system can be modeled with better accuracy since CALPHAD is an extrapolative technique. Moreover, the measured thermal conductivity values for liquid $\mathrm{Ga}$ from the present work matches well with the experimental data reported in the literature for similar temperatures. This again proves that the modified comparative cut bar technique is suitable for measuring thermal conductivities of liquid metallic alloys.

Figure 7 shows the comparison between the calculated and experimental thermal conductivities of the liquid phase in the Ga-In system. It is obvious from these results that most of the 
experimental data correlates satisfactorily well with the calculated values. Moreover, the thermal conductivity of the liquid Ga-In alloy at $\mathrm{w}(\operatorname{In})=0.3$ is the highest, while the values for the eutectic alloy and $w(I n)=0.1$ are similar. The comparison between the experimental thermal conductivities and calculated values in the $\mathrm{L}+\beta$ two-phase region is shown in Fig. 8. It was found that the extrapolation of the thermal conductivity parameters for the liquid and $\beta$ phases, in combination with the interface scattering parameters, can describe the experimental data well. It indicates that the thermal conductivities can be altered considerably due to the impediment by interfaces in the two-phase region. Hence, extrapolation from the individual phases alone will not suffice to reproduce the experimental thermal conductivity data satisfactorily using the CALPHAD method in the two-phase region. Besides, the thermal conductivity of the two-phase region is lower than the single-phase liquid. Nevertheless, it is higher than the highest reported thermal conductivity amongst the currently used TIMs. Thus, a set of self-consistent parameters for the thermal conductivity of pure elements, liquid, and $\mathrm{L}+\beta$ two-phase region of the Ga-In system has been developed successfully using the CALPHAD method with the input data from literature as well as measurements from the present work.

\section{Summary and conclusions}

In this work, the thermal conductivity of the Ga-In system was modeled using the CALPHAD method and compared with the experimentally measured values. Based on the analysis presented above, the significant outcomes from the present study can be summarized as follows.

- A modified comparative cut bar technique was used to demonstrate that the thermal conductivities of liquid and semi-liquid metallic alloys can be measured precisely. The error associated with this technique was $\sim \pm 1 \%$, which arises from the temperature sensor, unlike the $\pm 13 \%$ error in the thermal conductivity calculated from measurement of thermal diffusivity and density as well as estimation of specific heat capacity.

- The thermal conductivities of pure Ga in the liquid state, as well as liquid and $\mathrm{L}+\beta$ twophase region of the Ga-In system, were measured successfully using the modified comparative cut bar method. The measured values for the liquid and semi-liquid Ga-In alloys were higher than the highest reported thermal conductivity for commercial TIM, hence, making liquid Ga-In alloy as a potential TIM for consumer electronics. 
- CALPHAD method was used for developing a self-consistent set of thermal conductivity parameters successfully for the pure elements, liquid phase, and the $\mathrm{L}+\beta$ two-phase region of the Ga-In system with the available experimental data in the literature and the thermal conductivity values measured in the present work as input.

- There was a good correlation between the calculated and the measured thermal conductivities of pure In and $\mathrm{Ga}$ in liquid and solid states, and the experimentally measured values from the present work matched well with the values available in the literature for similar temperatures.

- Most of the experimental data match satisfactorily well with the calculated thermal conductivities of the liquid phase. There is a good correlation between the calculated and experimental thermal conductivities of the $\mathrm{L}+\beta$ phase, which indicated that the interface scattering factor is crucial for describing the thermal conductivity of the twophase region.

\section{Acknowledgments}

P.M. is thankful to Shady Side Academy for providing him the opportunity for a summer internship in the Department of Mechanical Engineering and Materials Science, Swanson School of Engineering, University of Pittsburgh. W.X. acknowledges funding from the National Science Foundation under award DMR - 1808082.

\section{References}

[1] J.P. Gwinn, R.L. Webb, Performance and testing of thermal interface materials, Microelectronics J. 34 (2003) 215-222. doi:10.1016/S0026-2692(02)00191-X.

[2] D.D.L. Chung, Thermal Interface Materials, J. Electron. Mater. 49 (2020) 268-270. doi:10.1007/s11664-019-07732-3.

[3] C. Zhang, Y. Du, S. Liu, Y. Liu, B. Sundman, Thermal conductivity of Al-Cu-Mg-Si alloys: Experimental measurement and CALPHAD modeling, Thermochim. Acta. 635 (2016) 8-16. doi:10.1016/j.tca.2016.04.019.

[4] L. Huang, S. Liu, Y. Du, C. Zhang, Thermal conductivity of the Mg-Al-Zn alloys: Experimental measurement and CALPHAD modeling, Calphad Comput. Coupling Phase Diagrams Thermochem. 62 (2018) 99-108. doi:10.1016/j.calphad.2018.05.011.

[5] D. Zhao, X. Qian, X. Gu, S.A. Jajja, R. Yang, Measurement techniques for thermal 
conductivity and interfacial thermal conductance of bulk and thin film materials, J. Electron. Packag. Trans. ASME. 138 (2016) 1-19. doi:10.1115/1.4034605.

[6] N. Saunders, A.P. Miodownik, CALPHAD (calculation of phase diagrams): a comprehensive guide, Elsevier, 1998.

[7] H. Lukas, S.G. Fries, B. Sundman, Computational thermodynamics: the Calphad method, Cambridge university press, 2007.

[8] A.E. Gheribi, P. Chartrand, Application of the CALPHAD method to predict the thermal conductivity in dielectric and semiconductor crystals, Calphad Comput. Coupling Phase Diagrams Thermochem. 39 (2012) 70-79. doi:10.1016/j.calphad.2012.06.002.

[9] J. Xin, L. Gan, L. Jiao, C. Lai, Accurate density calculation for molten slags in SiO2A12O3-CaO-MgO systems, ISIJ Int. 57 (2017) 1340-1349. doi:10.2355/isijinternational.ISIJINT-2017-070.

[10] B. Zhang, X. Li, D. Li, Assessment of thermal expansion coefficient for pure metals, Calphad Comput. Coupling Phase Diagrams Thermochem. 43 (2013) 7-17. doi:10.1016/j.calphad.2013.08.006.

[11] K. Hack, G. Wu, E. Yazhenskikh, T. Jantzen, M. Müller, A CALPHAD approach to modelling of slag viscosities, Calphad Comput. Coupling Phase Diagrams Thermochem. 65 (2019) 101-110. doi:10.1016/j.calphad.2019.02.001.

[12] T.J. Anderson, I. Ansara, The Ga-In (Gallium-Indium) System, J. Phase Equilibria. 12 (1991) 64-72. doi:10.1007/BF02663677.

[13] V.Y. Prokhorenko, V. V. Roshchupkin, M.A. Pokrasin, S. V. Prokhorenko, V. V. Kotov, Liquid gallium: Potential uses as a heat-transfer agent, High Temp. 38 (2000) 954-968. doi:10.1023/A:1004157827093.

[14] M.J. Assael, A. Chatzimichailidis, K.D. Antoniadis, W.A. Wakeham, M.L. Huber, H. Fukuyama, Reference correlations for the thermal conductivity of liquid copper, gallium, indium, iron, lead, nickel and tin, High Temp. - High Press. 46 (2017) 391416.

[15] R.W. Powell, M.J. Woodman, R.P. Tye, Further measurements relating to the anisotropic thermal conductivity of gallium, Br. J. Appl. Phys. 14 (1963) 432-435. 
doi:10.1088/0508-3443/14/7/314.

[16] N. Maraşli, S. Akbulut, Y. Ocak, K. Keşlioğlu, U. Böyük, H. Kaya, E. Çadirli, Measurement of solid-liquid interfacial energy in the In-Bi eutectic alloy at low melting temperature, J. Phys. Condens. Matter. 19 (2007). doi:10.1088/0953$8984 / 19 / 50 / 506102$.

[17] O. Redlich, A.T. Kister, Algebraic Representation of Thermodynamic Properties and the Classification of Solutions, Ind. Eng. Chem. 40 (1948) 345-348. doi:10.1021/ie50458a036.

[18] M. DeSorgo, Thermal interface materials, Electron. Cool. 2 (1996).

[19] C.-P. Chiu, V. Solbrekken, G. L. LeBonheur, Y.E. Xu, Application of phase-change materials in Pentium (R) III and Pentium (R) III Xeon/sup TM/ processor cartridges, in: Proc. Int. Symp. Adv. Packag. Mater. Process. Prop. Interfaces, 2000: pp. 265-270.

[20] R. Viswanath, V. Wakharkar, A. Watwe, V. Lebonheur, Thermal performance challenges from silicon to systems, Intel Technol. J. (2000).

[21] T. Ollila, Navigating the maze of thermal interface materials, Electron. Prod. Mag. (1999).

[22] B. Rauch, Understanding the performance characteristics of phase-change thermal interface materials, in: ITHERM 2000. Seventh Intersoc. Conf. Therm.

Thermomechanical Phenom. Electron. Syst., 2000: pp. 42-47.

[23] B. Jansson, Ph.D. thesis, Royal Institute of Technology, Stockholm, Sweden, 1984.

[24] B. Sundman, B. Jansson, J.-O. Andersson, The Thermo-Calc Databank System, Calphad. 9 (1985) 153-190. 


\section{Figure captions:}

Fig. 1. Schematic illustration showing the effectiveness of liquid TIM in filling the insulating air pockets for efficient heat conduction in comparison with the solid TIM applied between the mating surfaces i.e., surfaces of CPU and heat sink.

Fig. 2. Schematic of the setup used for measuring the thermal conductivity using (a) original and (b) modified comparative cut bar technique.

Fig. 3. Calculated Ga-In phase diagram using the thermodynamic descriptions from [12]. The black points indicate the temperatures and compositions at which the thermal conductivity of Ga-In alloys were measured in the present work.

Fig. 4. Schematic of the experimental setup used for measuring the thermal conductivity of pure Ga and Ga-In alloys using the modified comparative cut bar method.

Fig. 5. Comparison between calculated (solid line) and experimental (symbols) thermal conductivities of pure $\mathrm{Ga}$ in (a) liquid and (b) solid state.

Fig. 6. Comparison between calculated (solid line) and experimental (symbols) thermal conductivities of pure In in (a) liquid and (b) solid state.

Fig. 7. Comparison between calculated (solid line) and experimental (symbols) thermal conductivities of liquid Ga-In alloys at (a) $\mathrm{w}(\mathrm{In})=0.1$, (b) $\mathrm{w}(\mathrm{In})=0.214$ and (c) $\mathrm{w}(\mathrm{In})=0.3$.

Fig. 8. Comparison between calculated (solid line) and experimental (symbols) thermal conductivities of Ga-In alloy at $\mathrm{w}(\mathrm{In})=0.9$ in the $\mathrm{L}+\beta$ two-phase region.

\section{Table captions:}

Table 1. Thermal conductivity values measured using modified comparative cut bar technique for liquid and semi-liquid Ga-In alloys at different compositions and temperatures.

Table 2. Optimized thermal conductivity parameters for the individual phases and the two-phase regions in Ga-In system using the CALPHAD method. 
Table 1. Thermal conductivity values measured using modified comparative cut bar technique for liquid and semi-liquid Ga-In alloys at different compositions and temperatures.

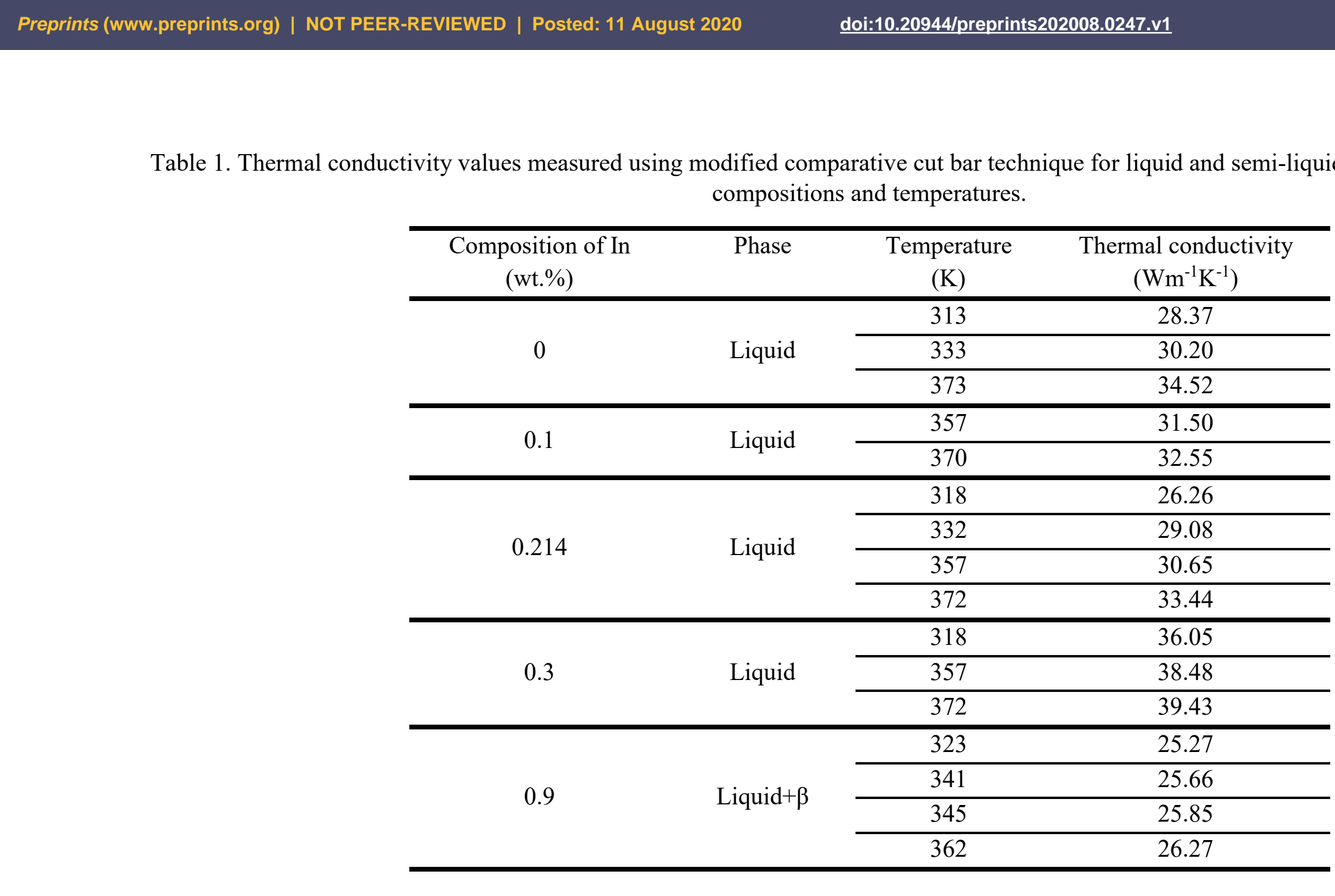

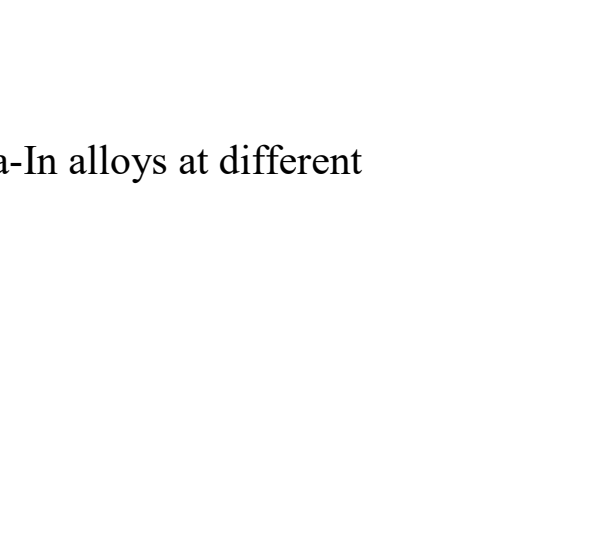

(1)

(

(1)

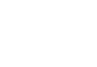

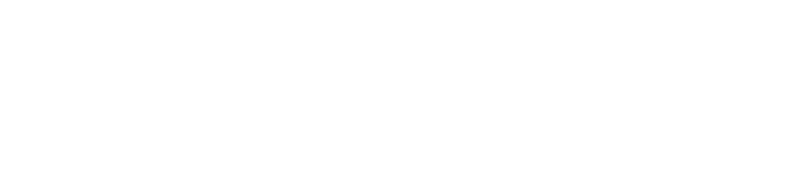

5


Table 2. Optimized thermal conductivity parameters for the individual phases and the two-phase regions in Ga-In system using the CALPHAD method.

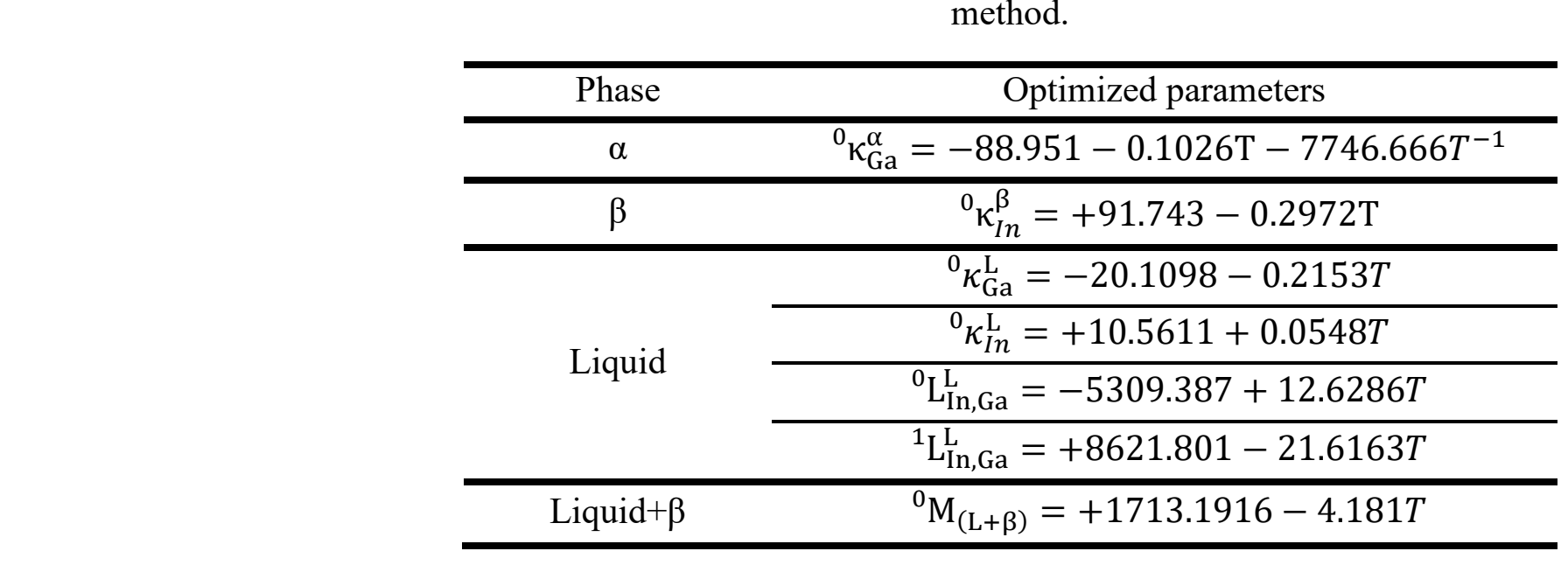

\section{oi:10.20944/preprints202008.0247.v1

16

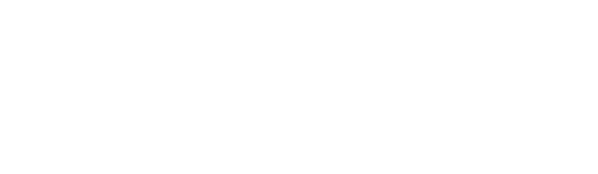

政




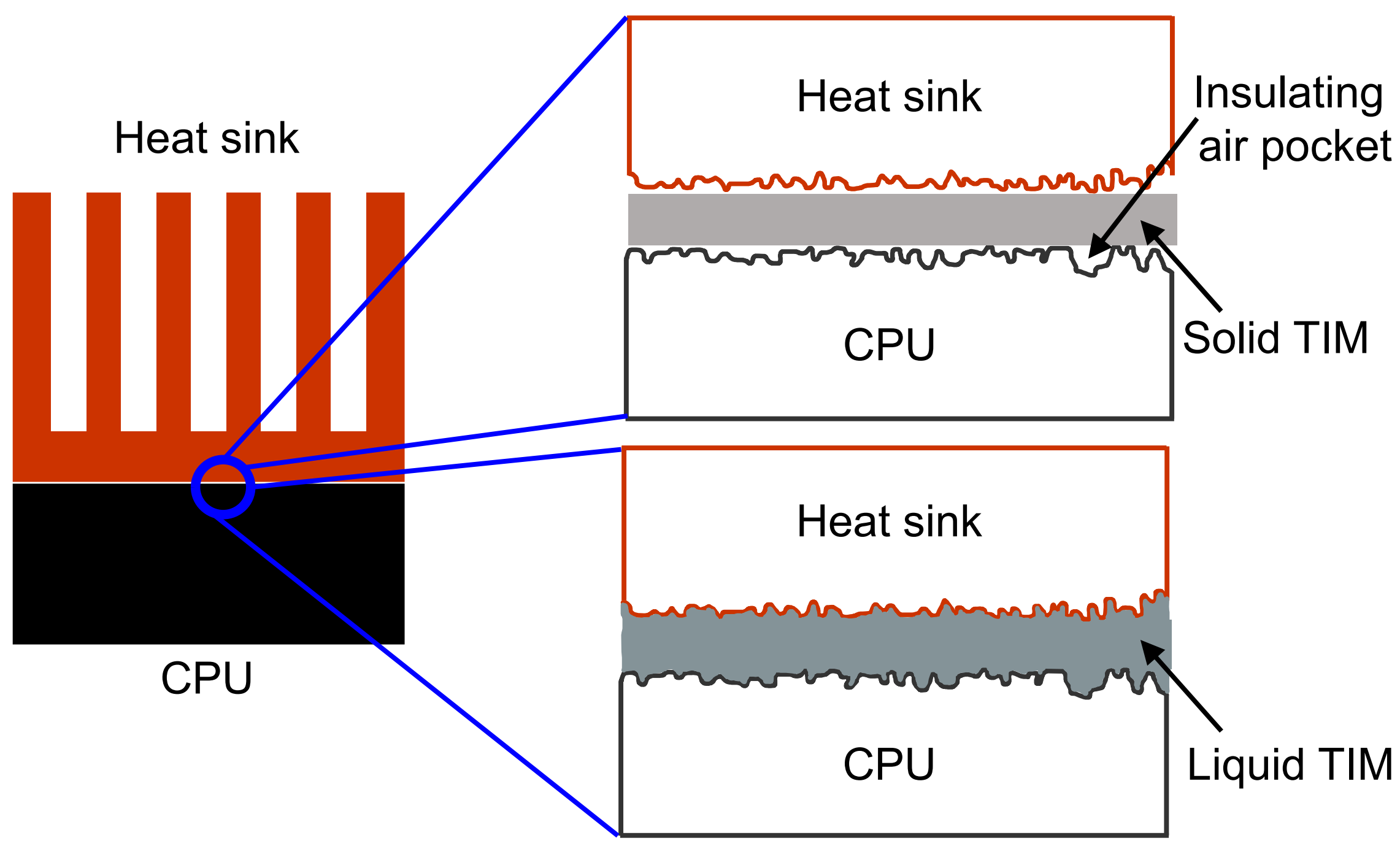

Fig. 1. Schematic illustration showing the effectiveness of liquid TIM in filling the insulating air pockets for efficient heat conduction in comparison with the solid TIM applied between the mating surfaces i.e., surfaces of CPU and heat sink. 


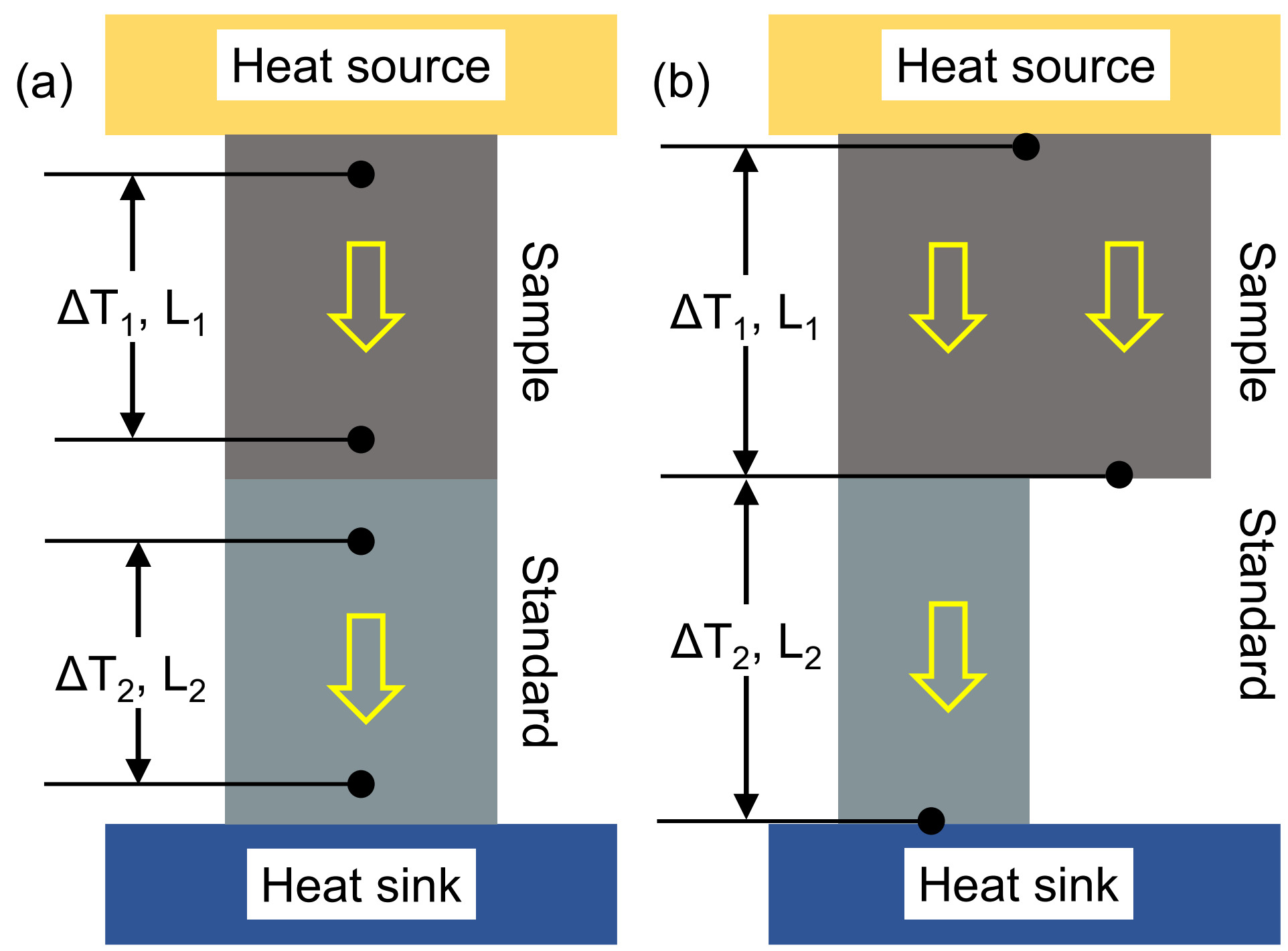

Fig. 2. Schematic of the setup used for measuring the thermal conductivity using (a) original and (b) modified comparative cut bar technique. 


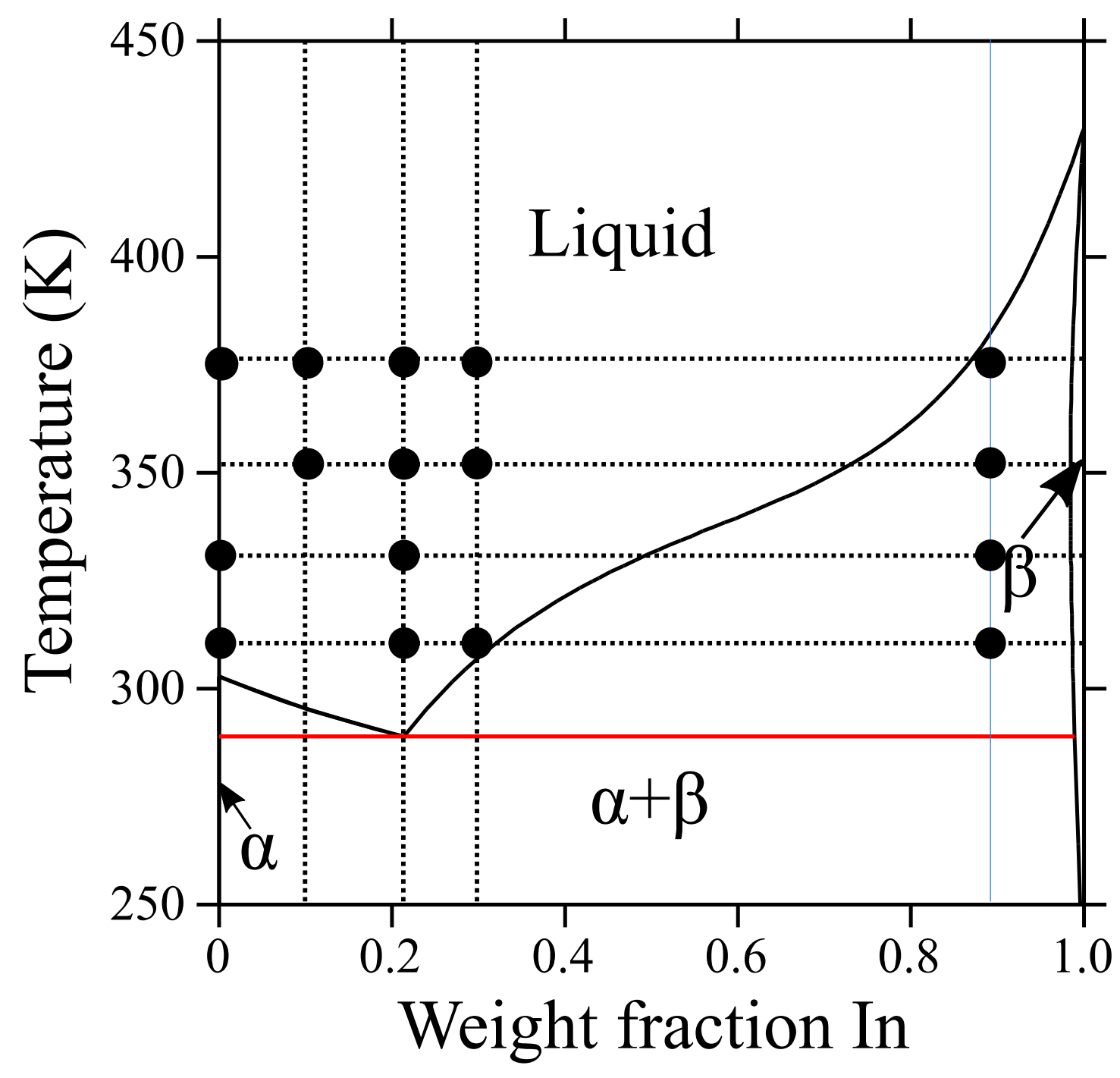

Fig. 3. Calculated Ga-In phase diagram using the thermodynamic descriptions from [12]. The black points indicate the temperatures and

compositions at which the thermal conductivity of Ga-In alloys were measured in the present work. 


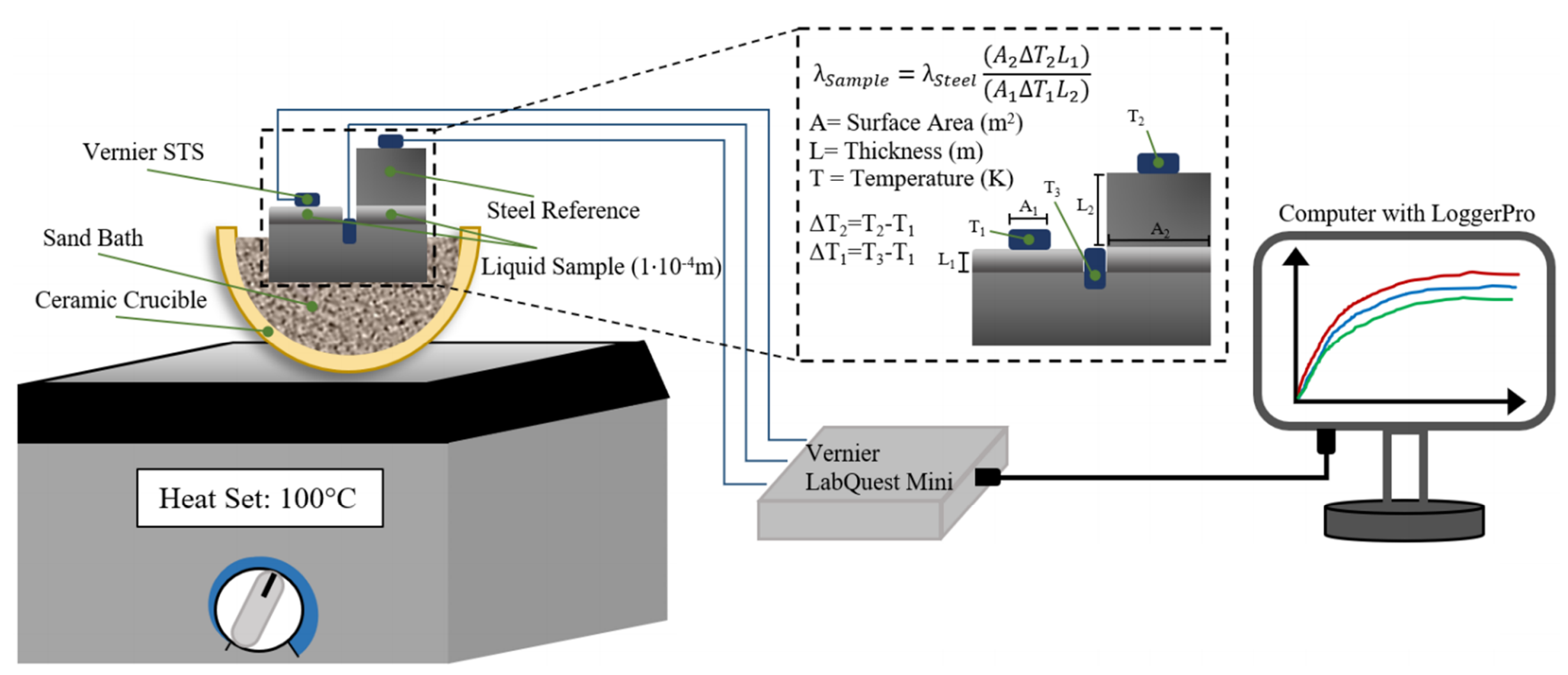

Fig. 4. Schematic of the experimental setup used for measuring the thermal conductivity of pure Ga and Ga-In alloys using the modified

comparative cut bar method. 

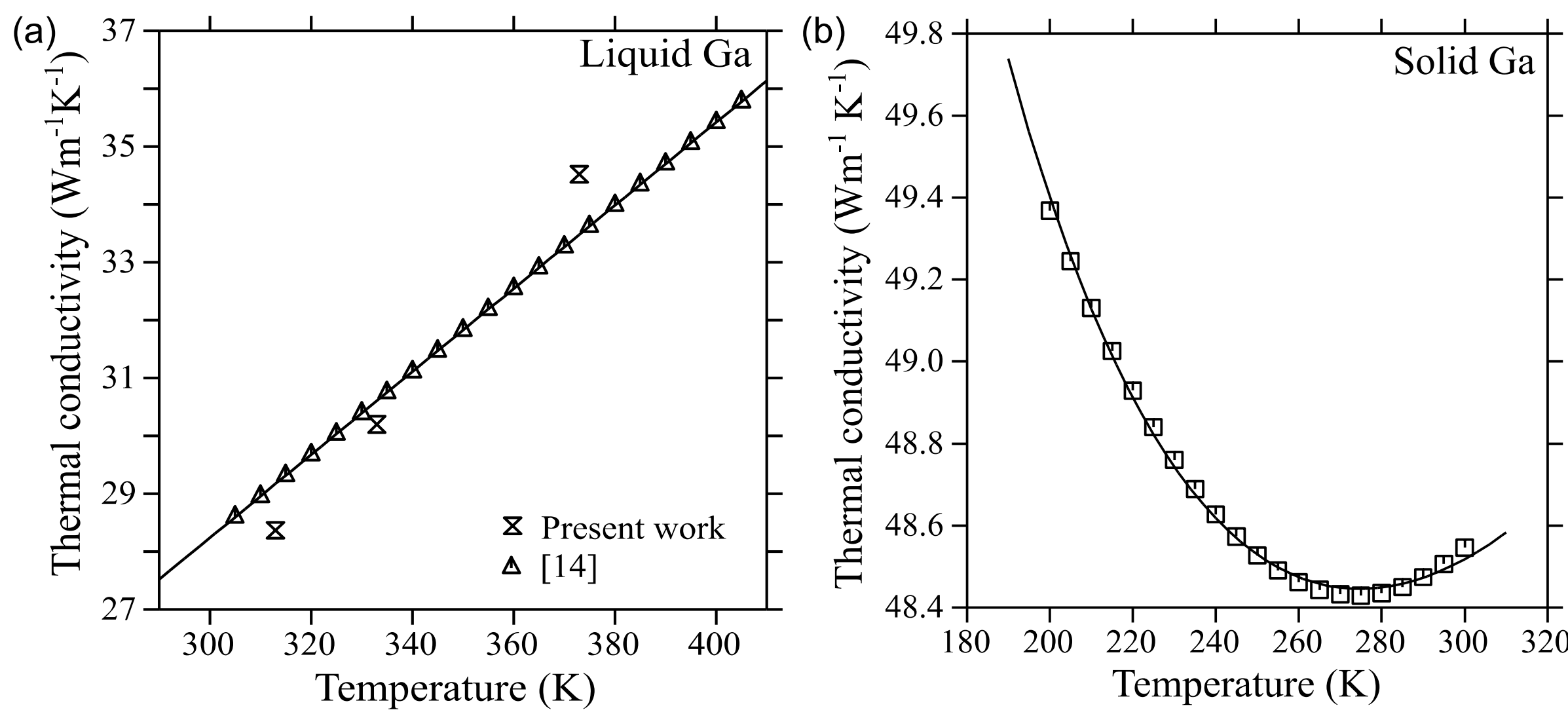

Fig. 5. Comparison between calculated (solid line) and experimental (symbols) thermal conductivities of pure Ga in (a) liquid and (b) solid state. 

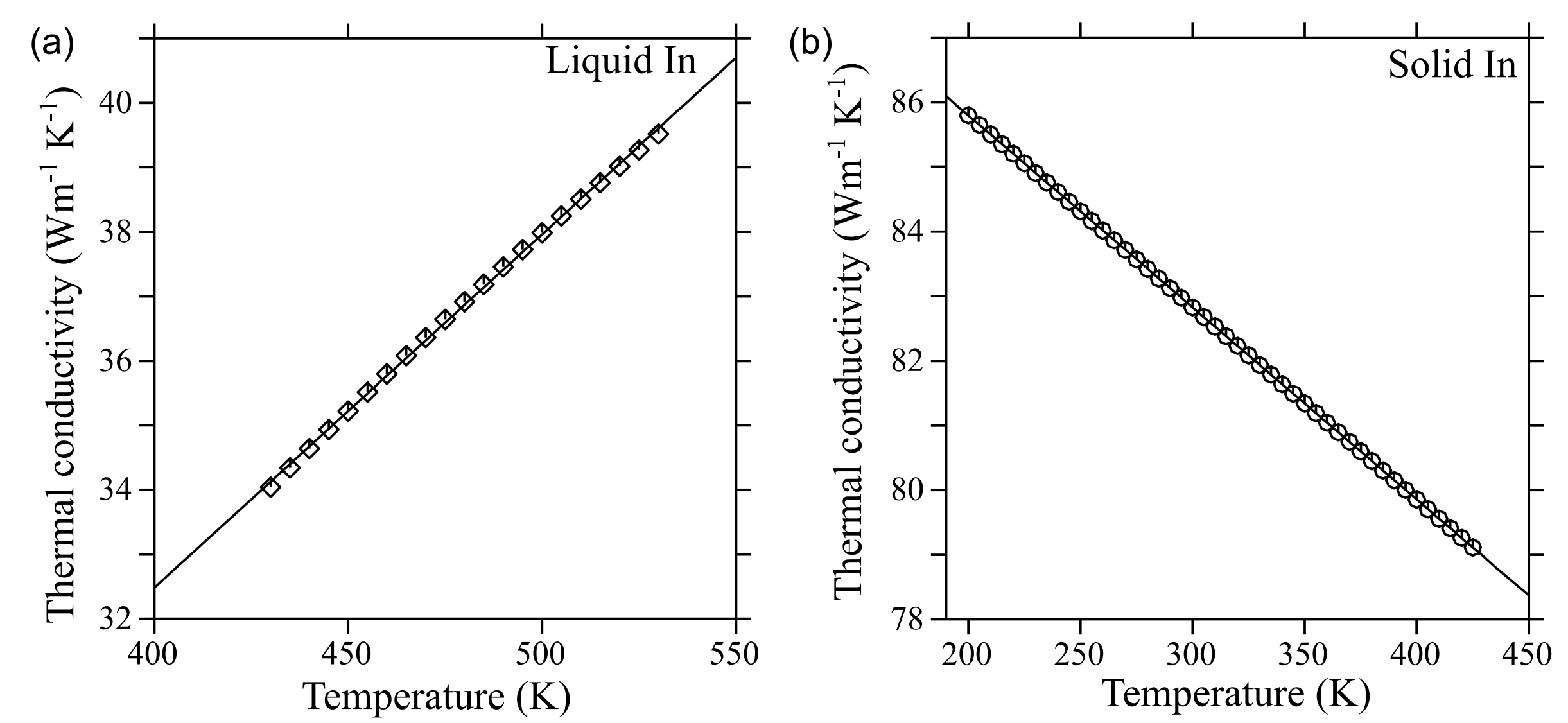

Fig. 6. Comparison between calculated (solid line) and experimental (symbols) thermal conductivities of pure In in (a) liquid and (b) solid state.

Fig. 6. Comprison betwen calculat (solid line) and expeim 

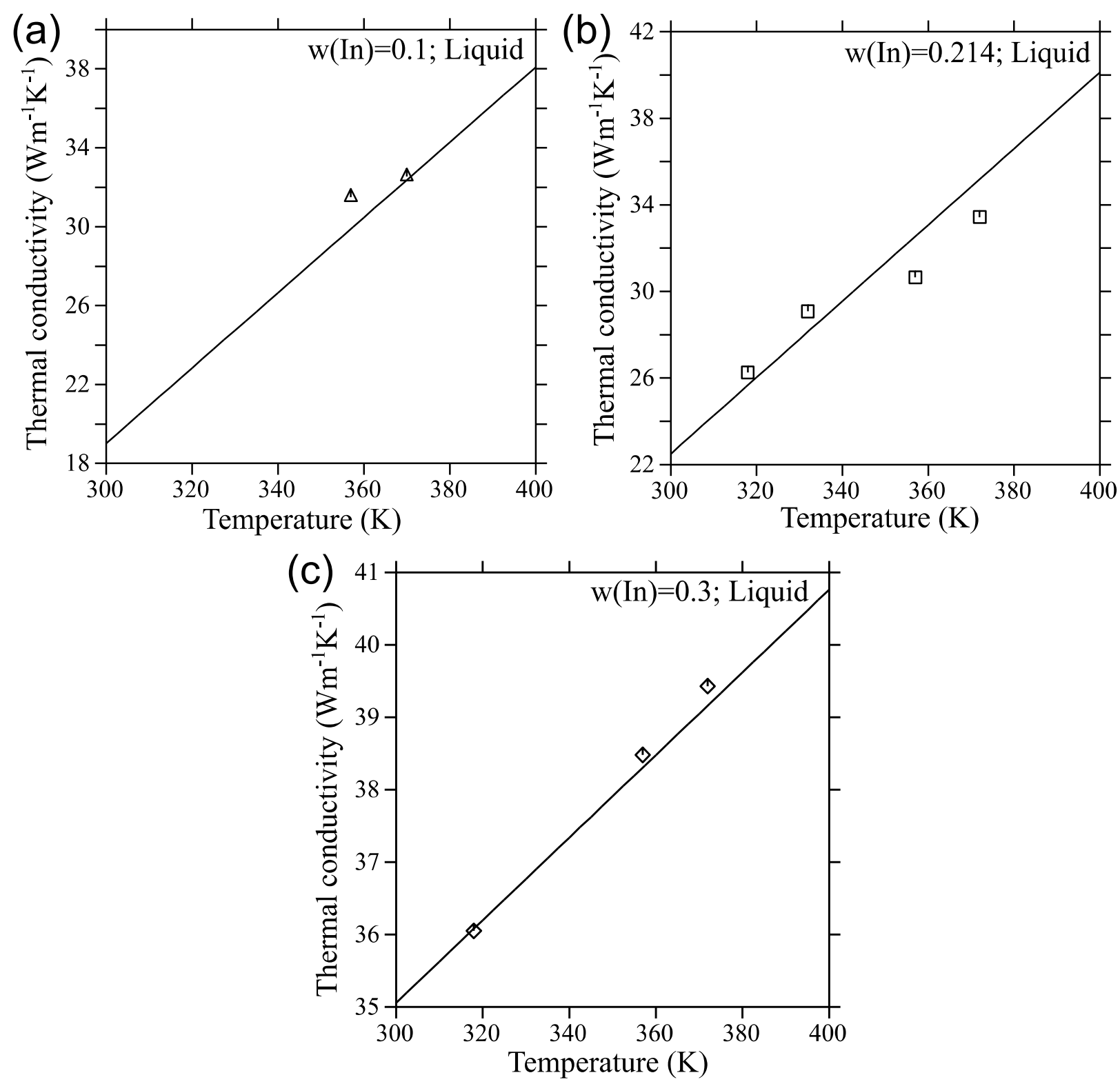

Fig. 7. Comparison between calculated (solid line) and experimental (symbols) thermal conductivities of liquid Ga-In alloys at (a) $\mathrm{w}(\mathrm{In})=0.1$, (b) $\mathrm{w}(\mathrm{In})=0.214$ and (c) $\mathrm{w}(\mathrm{In})=0.3$. 


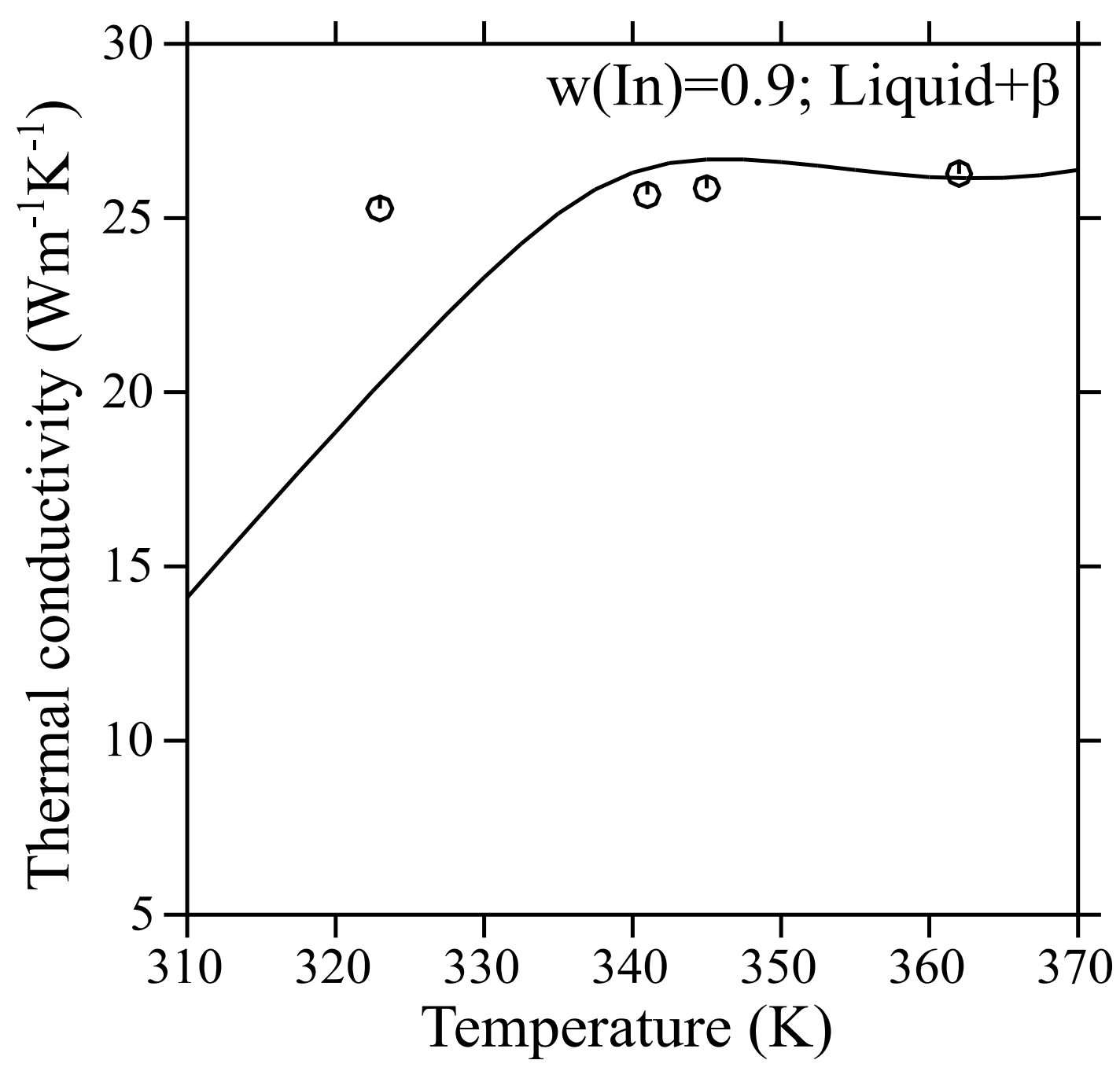

Fig. 8. Comparison between calculated (solid line) and experimental (symbols) thermal conductivities of Ga-In alloy at $w(\mathrm{In})=0.9 \mathrm{in}$ the $\mathrm{L}+\beta$

\footnotetext{
two-phase region.
} 